\title{
En el principio era la mano: Ernst Kapp y la relación entre máquina y organismo
}

\author{
In the beginning was the hand: Ernst Kapp and the relation between \\ machine and organism
}

\author{
Maurizio Esposito \\ Universidade Federal do ABC, Brasil / Universidad de Santiago de Chile, Chile \\ maurizio.esposito@ufabc.edu.br
}

\begin{abstract}
Resumen
La relación entre organismos y máquinas es muy antigua. Hace más de un siglo, el filósofo e historiador francés Alfred Victor Espinas señalaba que ya para los griegos la inteligibilidad de lo orgánico suponía una comparación con el mundo de la técnica. Aristóteles, por ejemplo, equiparaba los órganos con los artefactos mecánicos para entender el movimiento de los animales. En el período moderno, Descartes, Borelli y otros mecanicistas defendieron la idea que los organismos eran máquinas. Hoy en día, no faltan filósofos y científicos que consideran al genoma como un programa informático y al cerebro como un computador. En este artículo, pretendo reconsiderar la relación entre organismos y máquinas a partir de propuesta de un autor todavía poco conocido: el filósofo y geógrafo alemán Ernst Kapp (1808-1896), unos de los padres fundadores de la Filosofía de la Técnica y la Tecnología. Rompiendo con una larga tradición filosófica, Kapp defiende la idea que las máquinas son, en realidad, "proyecciones orgánicas". Los organismos no son máquinas, sino que, por el contrario, las máquinas son una imitación o reflejo del mundo orgánico. En primer lugar, aclararé la hipótesis de la proyección orgánica (así como sus alcances y límites), y, en segundo lugar, exploraré algunas de las consecuencias filosóficas de dicha hipótesis respecto al debate actual entre mecanicistas y anti-mecanicistas. Finalmente, defenderé la importancia de un acercamiento entre la Filosofía de la Tecnología y la Bio-filosofía para mejor entender el desarrollo de la Biología contemporánea.
\end{abstract}

Palabras clave: mecanismo, organismo, máquina, filosofía de la biología, filosofía de la tecnología.

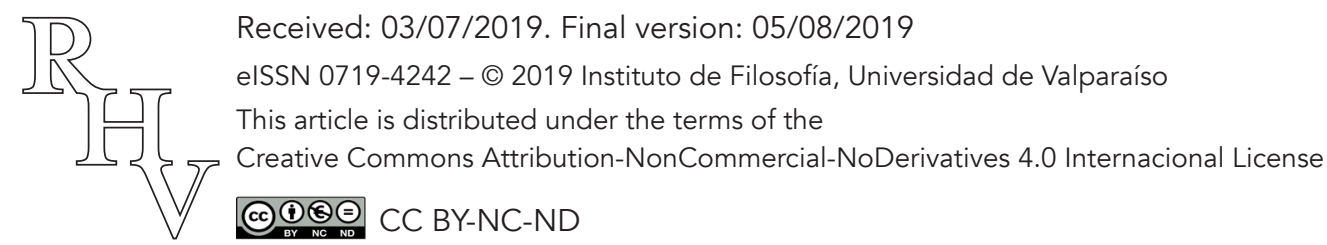


En el principio era la mano: Ernst Kapp y la relación entre máquina y organismo Maurizio Esposito

\begin{abstract}
The relation between organisms and machines is very old. Over a century ago, the French historian and philosopher Alfred Victor Espinas observed that from the Greeks onwards the intelligibility of the organic world presupposed a comparison with technical objects. Aristotle, for instance, associated living organs with mechanical artefacts in order to understand animals 'movements. In the modern period, Descartes, Borelli and other mechanists defended the idea that organisms are, in reality, machines. Today, philosophers and scientists still argue that the genome is like a software and the brain is like a computer. In this article I reconsider the relation between organisms and machines from the perspective of the German geographer and philosopher Ernst Kapp (1808-1896), one of the founding fathers of the Philosophy of Technology. Breaking with a long and venerable philosophical tradition, Kapp argued that machines are, in reality, "organic projections". Organisms are not machines; they are an imitation or reflection of the organic world. First of all, I clarify the hypothesis of "organic projection" (including its virtues and limits). Secondly, I consider some of the philosophical consequences that such a hypothesis entails over the debate between machinists and anti-mechanists. Finally, and following the previous considerations, I defend the importance of reconnecting the philosophy of technology with philosophy of biology in order to better understand the development of contemporary biology.
\end{abstract}

Keywords: mechanism, organism, machine, philosophy of biology, philosophy of technology.

\title{
1. Introducción
}

¿Qué es un organismo? ¿Qué es una máquina? Parece que no podemos responder a la primera pregunta sin considerar a la segunda. El filósofo e historiador francés Alfred Victor Espinas señalo que ya para los griegos la inteligibilidad de lo orgánico suponía la comparación con el mundo de la técnica. La ambigüedad de la palabra ópyavov - la cual denotaba ambos instrumentos artificiales o partes de un organismo - atestigua esta antigua afinidad. Aristóteles, por ejemplo, equiparaba los órganos a las máquinas estableciendo una conexión productiva entre la comprensión de los movimientos de los animales y los artefactos mecánicos (Espinas 1897; 1903). Además, en su tratado sobre el alma (Пعрì $\Psi v \chi \tilde{\eta} \varsigma$ ), Aristóteles consideraba los cuerpos vivos (soma organikon) como un conjunto de instrumentos (órganos) para el alma. ${ }^{1}$ Si bien los griegos establecieron un lazo significativo entre entidades biológicas y objetos técnicos, es solo en el periodo moderno que la relación entre máquinas y organismos se vuelve un problema filosóficamente urgente. Con René Descartes, Giovanni Borelli o Julien Offray de la Mettrie, así como también con los varios artesanos e ingenieros como Jacques de Vaucanson o Pierre Jaquet-Droz,

\footnotetext{
1 "Todos los cuerpos naturales, en efecto, son órganos del alma tanto los de los animales como los de las plantas [...]”, 2.4.415b18 (Aristóteles 2003).
}

Revista de Humanidades de Valparaíso, 2019, No 14, 117-138

(c) $(9)(9)$ CC BY-NC-ND 
En el principio era la mano: Ernst Kapp y la relación entre máquina y organismo Maurizio Esposito

la idea que pueda subsistir una continuidad profunda entre mundo vivo y artefactos se hizo muy persuasiva, aunque cuando la naturaleza especifica de dicha continuidad estaba lejos de ser clara (Duchesneau 1998; Wood 2002; Riskin 2007; Heudin 2008). Después de todo, ¿En qué sentido una máquina puede ser considerada una representación de una entidad orgánica? ¿Podemos decir que la máquina (autómata, reloj o herramienta) captura algunos elementos esenciales que caracterizan un organismo (ej. movimiento, fines, organización, etc.)? $\mathrm{O}$, ¿Que la máquina es una herramienta epistémico/instrumental que nos puede ayudar a comprender lo que es un ser orgánico en general $?^{2}$ Ahora, si consideramos la correlación entre artefactos mecánicos y organismos en su desenvolvimiento histórico, podemos rastrear por lo menos dos maneras fundamentales de compararlos $\mathrm{y}$, en la eventualidad, contrastarlos. La primera manera es la que llamo "mecanocéntrica" y la segunda es la que denomino tentativamente "organocéntrica".

Sin duda, la relación "mecanocéntrica" ha sido históricamente la más importante. Desde los griegos hasta la filosofía moderna y contemporánea, la idea que los organismos se "parecen" a las máquinas es la postura que ha recibido gran parte de la atención. Con relación "mecanocéntrica" me refiero a la idea que la representación que tenemos de un organismo posee una relación isomorfamente aproximada a la representación que tenemos de una máquina. Desde una perspectiva estructural y funcional, podemos decir que un organismo es una máquina muy compleja y la diferencia entre el primero y la segunda es solo de grado y no de calidad. Por tanto, en el ámbito de la relación "mecanocéntrica", tenemos posturas mecanicistas o anti-mecanicistas dependiendo si se enfatizan o restan los parecidos entre máquinas y seres vivos. A diferencia de la primera, la relación "organocéntrica" ha raramente suscitado un interés parecido, con la excepción de algunos notables autores que mencionaré en el presente artículo. Este tipo de relación parte de un presupuesto muy distinto respecto a la manera "mecanocéntrica", es decir, los organismos son máquinas solo en la medida que estas últimas imitan a los primeros. En otras palabras, la máquina, o los varios tipos de mecanismos artificiales son, en realidad, una proyección o extensión del mundo biológico. Los organismos, por tanto, no pueden ser considerados como máquinas porque estas últimas son un derivado mimético del mundo orgánico. En términos un poco más esquemáticos, podemos decir que de acuerdo a la relación "mecanocéntrica", el dominio de lo mecánico incluye el subconjunto de lo orgánico. Por el contrario, en la relación "organocéntrica" el dominio de las máquinas es un subconjunto del dominio del mundo orgánico en cuanto el organismo es el prototipo o modelo de cualquier máquina. En el primer caso, entonces, los organismos son, en verdad, máquinas o algo parecido. En el segundo caso las máquinas son, en principio, reducibles al mundo orgánico.

Quizás sorprenda que no haya muchos autores que exploraron seriamente esta segunda opción. Entre los más importantes, el más reciente es el filósofo francés Canguilhem y el

\footnotetext{
${ }^{2}$ Para un análisis filosófico más profundo relacionado a estas preguntas, ver Nicholson 2012.
} 
En el principio era la mano: Ernst Kapp y la relación entre máquina y organismo Maurizio Esposito

más antiguo es el geógrafo alemán Ernst Kapp. Canguilhem, dedica un breve y muy profundo ensayo al asunto: Machines et Organisme. En este ensayo, Canguilhem observaba que, en realidad, era la noción de organismo a definir la de mecanismo y no lo contrario: En enfatizando la ambigüedad esencial, y al mismo tiempo reveladora, del concepto griego de ópyavov, Canguilhem concluía aristotélicamente que las: "Herramientas y máquinas son órganos y los órganos son herramientas y máquinas" (1952). Por supuesto, Canguilhem retomó esta idea de interconexión entre tecnología y organismo desde Espinas y Espinas, a su vez, la había tomada de Ernst Kapp. ${ }^{3}$ Este último anticipó la hipótesis del origen orgánico de las máquinas en su magnus opus, Grundlinien einer Philosophie der Technik (Elementos de Filosofía de la Técnica), publicada en $1877^{4}$, unos de los primeros libros filosóficos dedicados al fenómeno de la técnica y tecnología.

En el presente artículo pretendo hacer tres cosas: en primer lugar, aclarar en la mejor manera posible la relación "organocéntrica" entre máquinas y organismos reconsiderando y analizando el trabajo de Kapp de 1877. En segundo lugar, explorar algunas de las consecuencias filosóficas que dicha relación implica examinando las siguientes preguntas: si las máquinas son una proyección e imitación de lo orgánico, como Kapp sostenía, ¿Qué pasa con la dicotomía tradicional mecanicismo/ anti-mecanicismo? Además, ¿cómo y en qué sentido un artefacto puede entregar conocimientos sobre entidades orgánicas? En tercer lugar, pretendo señalar algunos puntos de conexiones estratégicos entre la Filosofía de la biología y Filosofía de la tecnología y sugerir que, si aceptamos la hipótesis "organocéntrica", podemos llegar a la siguiente conclusión: los organismos son ontológicamente distintos de las máquinas y, sin embargo, éstas nos proporcionan conocimientos validos sobre diferentes aspectos del mundo orgánico. Esto significa que podemos ser ontológicamente escépticos respecto a la identidad entre organismo y máquina y, al mismo tiempo, ser confiados sobre al valor epistémico de las máquinas para conocer los fenómenos biológicos. En suma, la hipótesis de la proyección orgánica (relación organocéntrica) —la cual es el resultado de una conjunción eficaz entre bio-filosofía y tecno-filosofía - nos permite defender una postura anti-mecanicista a un nivel ontológico y mecanicista a un nivel epistémico.

\footnotetext{
${ }^{3}$ En los años que siguieron la publicación de Machines et Organisme, un estudiante de Canguilhem que desarrolló en forma extensa la relación entre técnica y organismo, Gilbert Simondon, solo considera a los trabajos de Espinas por medio de Canguilhem mientras que la fuente original se había en buena parte olvidado (Simondon 2012). De hecho, hay que considerar que, hasta donde alcanzo a conocer, la primera traducción en francés del opus magnus de Kapp, Grundlinien einer Philosophie der Technik, remonta a 2017 y la traducción en inglés es de 2018.

${ }^{4}$ Es cierto que a finales del siglo XIX y a lo largo del siglo XX las ideas "organocéntrica" se desarrollan en diferentes maneras de acuerdo a varios autores. Podemos recordar, entre otros y aparte Espinas y Canguilhem, el antropólogo Arnold Gehlen (1989), el filósofo José Ortega y Gasset (1965) e incluso el controversial historiador Oswald Spengler (2015). Un autor más reciente que reconsidera la tradición "órganocéntrica" es Hacking (1998).
}

Revista de Humanidades de Valparaíso, 2019, No 14, 117-138

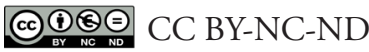




$$
\begin{gathered}
\text { En el principio era la mano: Ernst Kapp y la relación entre máquina y organismo } \\
\text { Maurizio Esposito }
\end{gathered}
$$

\section{Desde los órganos a las máquinas}

Ernst Kapp nace en Ludwigstadt, Bavaria, en 1808. Tal como el filósofo de Trier, Karl Marx, Kapp es un asiduo lector de Hegel y se orienta hacia la corriente de la izquierda hegeliana. En principio Kapp estudia filología clásica en la Universidad de Bonn. Luego se dedica a desarrollar un materialismo filosófico dirigido a integrar la nueva disciplina de la geografía (que Carl Ritter había fundado en la Universidad de Berlín) con la historia humana. En particular, Kapp es interesado a como el medio ambiente pueda plasmar y fomentar distintas formaciones socio-culturales (Mitcham 1994). En 1849, como consecuencia de un proceso por sedición relacionado a la publicación de un ensayo ${ }^{5}$ en contras del despotismo político, Kapp escapa en los Estados Unidos. Llega en Sisterdale, una pequeña colonia alemana en Texas. El ambiente de la frontera estadunidense, junto a su actividad de agricultor, fomenta su interés sobre la relación entre máquinas, herramientas y anatomía humana. En 1865 vuelve a Alemania y se dedica nuevamente al trabajo académico. Su Grundlinien del 1877 es una síntesis de sus estudios de geografía comparada y sus reflexiones sobre la experiencia americana (Mitcham 1994). El texto presenta una teoría muy ambiciosa sobre la relación entre biología y tecnología, y, por tanto, una hipótesis ingeniosa sobre las conexiones entre órganos y máquinas, considerando estas últimas como una "derivación" de los primeros.

De hecho, en contras de la tradición Cartesiana, Kapp era muy enfático en sostener que no había que confundir las máquinas con los organismos. Si bien podemos identificar varios elementos de similitud - entre otros, organización, movimiento, estructura y función-, las dos entidades, así como los conceptos de mecanismo y orgánico, refieren a dominios distintos. Confundir el concepto de máquina con el concepto de organismo significaba, por Kapp, no entender la verdadera relación entre los dos. Como primera aproximación, podemos decir que, desde la perspectiva de Kapp - y de varias posturas "organocéntricas" posteriores - asimilar el organismo a la máquina sería equivalente en asimilar un retrato de Luis XVI con el rey Luis XVI. Hay seguramente un parecido entre el rey y su retrato, sin embargo, no podemos concluir que el retrato es, en realidad, el rey. Si aplicamos el mismo razonamiento criticando la postura mecanicista, podemos argumentar que el parecido de una máquina a un organismo no sugiere una verdad sobre el estatus ontológico de los organismos (ej. Una identidad entre la primera con el segundo), sino señala algo sobre las producciones y representaciones humanas respecto a ciertas entidades naturales. Es decir, los artefactos mecánicos no son ontológicamente asimilables a algunos principios básicos de la organización biológica, sino que son una derivación de estos últimos, así como un retrato es una derivación o "proyección" del individuo Luis XVI. La relación entre entidad y su derivado es lo que Kapp llama "proyección orgánica”.

\footnotetext{
${ }^{5}$ El articulo incriminado era: "Der konstituierte Despotismus und die konstitutionelle Freiheit" ("Constituted despotism and constitutional liberty").
}

Revista de Humanidades de Valparaíso, 2019, No 14, 117-138

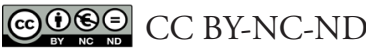


Para entender la naturaleza de la "proyección orgánica" y apreciar la novedad la postura de Kapp respecto a la relación máquina/organismo, tenemos que adentrarnos en algunos asuntos históricos, culturales y epistemológicos que caracterizan su propuesta. Empezando con los asuntos históricos, Kapp sostiene que la fabricación y uso de artefactos representa el verdadero inicio de la historia humana. En el primer capítulo de su Grundlinien, Kapp explica que la alienación inicial entre el ser humano (como ser orgánico entre otros) y el mundo externo queda superada a partir de la mediación de las actividades manuales. Mientras que los animales perciben el mundo externo sin tener la posibilidad de comprenderlo, los seres humanos lo comprenden en la medida que lo manipulan. Y en virtud de esta interacción práctica, el ser humano es el único organismo que logra ir más allá del puro dado empírico. En otras palabras, para los organismos no humanos, el mundo externo es un mundo de fenómenos caóticos, un conjunto de acontecimientos sin un sentido particular; un revoltijo de eventos que el organismo debe simplemente sufrir y tolerar para sobrevivir. En este caso, la relación con el mundo es puramente instintiva. Por lo contrario, en el caso del animal humano, la asimilación es posible en la medida que el organismo no solo percibe los fenómenos, sino que los controla, los domina y los predice. Este control y dominio de los fenómenos no puede ser algo abstracto o teórico (contemplativo), sino algo esencialmente concreto y práctico: los seres humanos asimilan (comprenden) el mundo externo por medio de sus intervenciones y manipulaciones. Inicialmente este proceso practico se orienta hacia los objetos naturales presentes en el entorno (piedras, huesos, troncos de madera etc.), después y progresivamente, la atención se mueve hacia la fabricación de artefactos. La verdadera integración con el medio ambiente, y lo que diferencia la prehistoria de la historia, es la articulación intencional de objetos funcionales.

Ahora, Kapp no sostiene que los seres humanos son meros primates que fabrican herramientas. Si así fuese, deberíamos extender esta capacidad a muchos otros organismos. La diferencia esencial entre los humanos y no humanos no se reduce a la mera fabricación de utensilios sino en la consciencia que los seres humanos adquieren por medio de esta actividad. De hecho, Kapp sostiene que la producción de artefactos es un factor evolutivo donde el ser humano aprende sobre su entorno y sí mismo. La autoconsciencia es un producto de una reflexión sobre el trabajo de las manos. Hacer y manejar utensilio es una condición esencial para la emergencia del pensamiento reflexivo. Esto implica que la autoconsciencia no es un fenómeno puramente mental, sino un fenómeno que emerge a partir de la reiteración productiva de actividades prácticas. La autoconsciencia es un producto de la mente en un cuerpo y no un derivado etéreo del cuerpo. En este sentido, mientras que los animales plasman y utilizan herramientas por medio de instintos específicos, los seres humanos producen a partir de una reflexión o actividad concreta que es genuinamente creativa. Kapp explicita la relación entre consciencia y actividad práctica reconstruyendo la etimología de la palabra alemana selbst, el cual sería un término compuesto de si y liba, es decir, "vida" y "miembros". La palabra misma nos indica que la 
En el principio era la mano: Ernst Kapp y la relación entre máquina y organismo Maurizio Esposito

consciencia de sí mismo, lo que los anglosajones llaman self, es el efecto de la interacción entre acción y reflexión, concreción y abstracción, estructura y función, cognición y cuerpo. Más allá de la veracidad etimológica de selbst, Kapp nos quiere convencer de dos premisas fundamentales que están a la base de su argumento de la "proyección orgánica": 1) Los artefactos son al mismo tiempo, herramientas de adaptación y herramientas de comprensión. 2) La historia humana comienza con la práctica, con la fabricación y uso de utensilios en la medida que la praxis y manejo de instrumentos es la condición esencial para le emergencia de la autoconsciencia. Como Kapp argumenta:

Los animales también tienen una percepción sensible inmediata de las cosas. Empero, el animal no entiende lo que observa y escucha, come o huele; estas cosas permanecen distantes o alienas a su ser, como una antítesis en la cual el animal persiste. El ser humano, por el contrario, supera esta antítesis en virtud de su disposición original que le confiere la competencia en expandir ad infinitum, en forma productiva y receptiva, sus habilidades sensoriales que comparte con los otros animales por el medio de suportes mecánicos - en otras palabras, por el medio del trabajo de sus manos. (Kapp 2018, 23)

No entendemos el sentido de la proyección orgánica si no entendemos que el ser humano es, en su esencia, un ser productivo y al mismo tiempo reflexivo. Y es precisamente la dialéctica entre producción y reflexión que define el proceso "proyectivo" de algunos elementos internos, subjetivo, hacia el exterior. El inicio de la historia (por medio de la acción) y el comienzo de la consciencia (por medio de la reflexión concreta sobre la producción) son coextendidos con la proyección orgánica.

Pero ¿en qué consiste, en lo específico, dicha "proyección”? Como primera aproximación, Kapp entiende con "proyección" la acción de ejecutar o explicitar algunos atributos subjetivos interiores hacia el exterior. Representaciones como un cuadro o una estatua pueden ser consideradas como "proyecciones" en la medida que estas se proyecten, en un soporte material, algunas imágenes subjetivas. Una obra de arte es entonces una "proyección" de un estado interior. Ahora, si bien haya una relación entre representación y proyección, Kapp sostiene que la proyección sea una actividad más fundamental, desde un punto de vista evolutivo y cultural, de la misma representación. El ser humano "proyecta" o "extrínseca" sus facultades cognitivas en objetos concretos y materiales cada vez que interactúa productivamente con la realidad. El acto fundacional epistémico que caracteriza la relación entre organismos humanos y medio ambiente reside, para Kapp, en la interacción práctica y concreta entre sujetos y mundo externo por medio de órganos/herramientas. La ambigüedad entre el concepto de órgano y el concepto de herramienta no es casual. Si el órgano/herramienta primitivo es la mano, el primero órgano/herramienta artificial es el martillo. La mano es el órgano/herramienta innato universal y es el prototipo de todos los otros órganos/herramientas artificiales (a partir del martillo). La mano (como recordaba Aristóteles en su Acerca del Alma), es el órgano de los órganos y el martillo es una "proyección orgánica" de la mano (y del brazo, como veremos). A su vez,

Revista de Humanidades de Valparaíso, 2019, No 14, 117-138

(c) (i) $(9)-\mathrm{O}$ CC BY-NC-ND 
En el principio era la mano: Ernst Kapp y la relación entre máquina y organismo Maurizio Esposito

el martillo es el órgano/herramienta prototípico de muchos otros órganos/herramientas artificiales. No hay límites a la cantidad de proyecciones orgánicas posibles que pueden surgir (y que de hecho surgieron) a parir de la articulación entre mano y martillo:

De esta manera, una abundancia de creaciones emerge a partir de la fuerza productiva humana por medio de la mano, brazo y diente. Un dedo torcido se vuelve un gancho, el hueco de la mano se trasforma en un recipiente. En una espada, una lanza, un remo, una pala, un rastrillo, arado, horquilla, podemos fácilmente rastrear la tendencia dinámica del brazo, de la mano y de los dedos, así como sus adaptaciones a las actividades de caza, pesca, siembre y cosecha. (Kapp 2018, 38)

En suma, todos los primeros artefactos (órganos/herramientas artificiales) son proyecciones de órganos/herramientas innatos (órganos del cuerpo). La misma historia humana nace con el martillo, el cual consiste en la "proyección orgánica" más importante en términos históricos y fisiológicos. El martillo, en conjunción con la mano, es el prototipo de todas las otras proyecciones orgánicas más complejas; desde una espada hasta la máquina a vapor. ${ }^{6}$

La producción de artefactos tiene entonces un valor filogenético. De hecho, Kapp sostiene que las manos, los brazos, así como todo el cuerpo co-evoluciona con los mismos artefactos y herramientas. Es decir, una herramienta se vuelve progresivamente un órgano/herramienta en la medida que un órgano innato se adapta a la herramienta artificial. Si bien con el proceso de co-evolución se realiza una articulación siempre más profunda entre órgano y herramienta, la evolución de los artefactos y de las máquinas adquieren, con el tiempo, una cierta autonomía. En la evolución de los martillos, espadas, rastrillos etc., la proximidad con los órganos humanos se mantiene. Con la evolución de los molinos, máquinas a vapor, telégrafos o locomotoras la proximidad se pierde, aunque el lazo con el organismo creador nunca se rompa. Kapp enfatiza que por cuanto una tecnología pueda ser sofisticada y por cuanto una máquina pueda perder cualquier semblanza con la fisiología humana o animal, todos los artefactos tienen su último origen en una proyección. Un descendiente de una proyección orgánica es a su vez una proyección orgánica.

\footnotetext{
${ }^{6}$ Hoy en día un buen ejemplo es el computador.
}

Revista de Humanidades de Valparaíso, 2019, No 14, 117-138

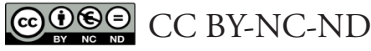


En el principio era la mano: Ernst Kapp y la relación entre máquina y organismo Maurizio Esposito

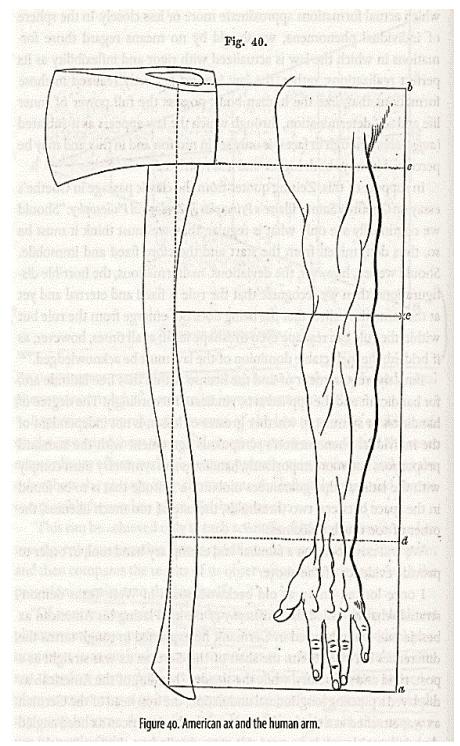

Fig. 1. Relación "proyectiva” entre un brazo y un hacha (Kapp 1877, 174).

Unos de los aspectos más interesantes de la teoría "organocéntrica" de Kapp es que la relación entre máquina y organismo no se basa sobre un isomorfismo explicito, sino sobre un lazo diacrónico y, al mismo, tiempo, practico. Me refiero al lazo diacrónico en la medida que existe una filogenia de proyecciones que tienen su antepasado común en el brazo y la mano y me refiero a un lazo práctico en la medida que los artefactos, las herramientas y después las máquinas, extienden o intensifican el trabajo humano remplazándolo. La necesidad fomenta la producción de una herramienta. La práctica y el trabajo estimulan el perfeccionamiento de la herramienta. El conjunto entre necesitad y practica incentiva el desarrollo de un artefacto tecnológico. Sin embargo, la relación virtuosa entre organismo y máquina no se reduce a una estrategia pragmática de integración con el medio ambiente. La máquina misma, como "proyección orgánica", es también y sobre todo lo que llamo un "dispositivo epistémico", es decir, una herramienta que produce comprensión. Para entender como un artefacto se vuelve un dispositivo que produce conocimiento del entorno, hay que mencionar algunos elementos importantes que rodean la hipótesis de la "proyección orgánica" de Kapp.

La teoría "organocéntrica" de Kapp presupone un momento inconsciente y un momento consciente respecto a la proyección. Mientras que inicialmente un artefacto es una proyección "inconsciente" de lo orgánico, en un segundo momento este mismo artefacto se vuelve un dispositivo que conscientemente nos entrega explicaciones y comprensión del mundo orgánico. Un aparato óptico no solamente amplia la visión, sino que también nos permite de entender la visión misma. Un aparato acústico no solamente amplifica nuestro oído, sino que también nos permite entender o representar la fisiología del oído. Una má- 
En el principio era la mano: Ernst Kapp y la relación entre máquina y organismo Maurizio Esposito

quina a vapor no solamente incrementa nuestra fuerza, sino que nos permite representar la misma naturaleza de la fuerza y del trabajo. Un cable del telégrafo no solamente extiende nuestras posibilidades de comunicar, sino que también nos entrega comprensión respecto a un cordón nervioso (ver Fig. 2 en la siguiente sección). En breve, el fenómeno de la proyección orgánica se puede dividir en tres momentos fundamentales: en primer lugar, hay la producción inconsciente de un artefacto que amplifica una función orgánica. En segundo lugar, hay el manejo del artefacto que lleva el sujeto a realizar, en forma consciente, que este último es una proyección de un órgano. Y, en tercer lugar, sobreviene la intuición que el artefacto es un dispositivo que genera comprensión respecto al mismo órgano y organismo. En la próxima sección veremos más en detalles como las proyecciones orgánicas, los artefactos y mecanismos, se vuelven dispositivos epistémicos confiables a partir de la dialéctica entre lo inconsciente y consciente.

\section{Repensar las máquinas a partir de los organismos}

En 1872, el historiador alemán, Alfred Wilhelm Dove, señaló que: “...entendemos el mecanismo de la naturaleza (fisiología del cuerpo) solo después que nosotros lo hemos libremente inventado - entendemos el ojo después de la invención de la cámara, así como entendemos los nervios después de la invención del telégrafo" (Dove, citado en Kapp 2018, 109). Kapp sigue detenidamente la intuición de Dove con una diferencia esencial; la producción de un artefacto no es algo puramente creativo (en un sentido de creación ex nihilo), sino una proyección inconsciente que, luego, se vuelve una herramienta de comprensión. Kapp explora una serie de ejemplos específicos para mostrar como un artefacto pueda funcionar como dispositivo epistémico. El considera aparatos ópticos (telescopio, microscopio, camera obscura etc.), infraestructuras (puentes), máquina a vapor (trenes), y el telégrafo (en particular la tecnología de los cables). Mi intención no es aquí comentar en detalle el texto de Kapp, sino subrayar algunos elementos que nos puedan ayudar a entender la lógica de la proyección orgánica y su relevancia actual.

Empezamos con la invención y desarrollo de los aparatos ópticos. ¿Cuál es la diferencia entre un ojo y una camera obscura? ¿Podemos sostener, de acuerdo a la hipótesis "mecanocéntrica", que el primero se puede reducir a la segunda? Kapp argumenta que la relación entre el órgano y el artefacto no es de "reducción" (ej. el ojo no es nada más que una especie de camera obscura), sino de "proyección" (ej. La camera obscura es una externalización inconsciente de un ojo, así como una lente del aparato es una proyección del cristalino del ojo). El hecho de que haya una relación de proyección inconsciente no implica que exista una identidad (mecanocéntrica) entre el aparato y el ojo. Lo que la relación realmente indica es que el inventor se ha inspirado, en forma inconsciente, a una entidad orgánica para construir un artefacto. Esto significa que no es el ojo que se parece a una camera obscura, sino que es esta última que se constituye a partir del primero. La relación "mecanocéntrica" se fundamenta sobre una relación "organocéntrica". En este

Revista de Humanidades de Valparaíso, 2019, No 14, 117-138

(c) $(9)(9)$ CC BY-NC-ND 
En el principio era la mano: Ernst Kapp y la relación entre máquina y organismo Maurizio Esposito

sentido, podemos sostener que el fenómeno de la proyección orgánica no es nada más que un proceso de bio-mimetismo involuntario. Y es precisamente en virtud de esta relación "biomimética" involuntaria que el aparato óptico se trasforma en un dispositivo epistémico. Una camera obscura, un telescopio o un microscopio no solamente nos permiten de fijar imágenes, engrandar los objetos lejanos o pequeños; ellos nos revelan los principios ópticos de la visión:

El misterio de la fisiología de este órgano (ojo) puede ser resuelto solo después que haya sido "proyectado" en diferentes números de aparatos mecánicos; así estableciendo una referencia externa para su propia construcción anatómica. El ser humano ha aprendido desde el instrumento que el mismo ha constituido a partir del órgano/herramienta óptico y los llevó a la comprensión del mecanismo de foco en relación a la luz refracta en el ojo hasta las lentes. (Kapp 2018, 62)

El mismo razonamiento se puede aplicar a otros artefactos y tecnologías, aunque la relación de "proyección” pueda manifestarse en diferentes formas. Por ejemplo, ¿Cuál puede ser la relación entre una máquina a vapor y un organismo? ¿En qué sentido una máquina a vapor manifiesta una similitud estructural o funcional con un cuerpo orgánico? Para los fisiólogos alemanes Hermann von Helmholtz y Robert von Mayer, la relación entre una máquina a vapor y un organismo se establece a nivel de movimiento y trabajo. Si nos preguntamos cual es el verdadero origen del movimiento y trabajo de una entidad cualquiera, la máquina a vapor podía servir como un dispositivo epistémico muy eficaz. Kapp justamente nos dirige hacia aquellos textos donde los dos fisiólogos reconocen una continuidad "organocéntrica" entre máquina y organismo. Helmholtz, en su ponencia "Uber die Wechselwirkung der Naturkrafte und die darauf bezuglichen neuesten Ermittelungen der Physik" (Sobre la interacción de las fuerzas naturales...), observaba que por medio de la máquina a vapor los fisiólogos habían esclarecido las orígenes del movimiento y del trabajo de un órgano/herramienta: "El cuerpo del animal no se diferencia, por tanto, de la máquina a vapor a partir de la manera de como produce calor y trabajo, sino de los propósitos y las maneras en que este calor y trabajo es aplicado" (Helmolthz, citado por Kapp 2018, 97). En otras palabras, tenemos fines, materias y estructuras distintas, sin embargo lo que une un organismo a una máquina a vapor comparten es su manera de producir calor y movimiento. Antes que Helmolthz formulara el principio de conservación de la energía en 1847, von Meyer había observado que los nutrientes son combustibles que generan calor, y por tanto movimiento y trabajo. Que sea carbón para la máquina o nutrientes para el organismo, todos se reducen por último a la energía solar acumulada y conservada en los vegetales. Por tanto, la construcción y funcionamiento de una máquina a vapor nos revela algunos de los principios básicos y simples que animan el movimiento orgánico. La máquina a vapor puede ser considerada dispositivo epistémico en virtud de una proyección orgánica que antecedió y fomentó el mismo desenvolvimiento de esta tecnología. Por tanto, no son los organismos que se reducen a una máquina, sino son las

Revista de Humanidades de Valparaíso, 2019, No 14, 117-138

(c) $(9)(9)$ CC BY-NC-ND 
En el principio era la mano: Ernst Kapp y la relación entre máquina y organismo Maurizio Esposito

máquinas que deben vincularse con el mundo orgánico. Se puede considerar la máquina a vapor un "organismo" solo en la medida en que la primera imita algunos elementos básicos que caracterizan los seres vivos.

Otro ejemplo que Kapp menciona es la tecnología del telégrafo. Poco más de 30 años antes que Kapp publicara su Grundlinien, Samuel Morse pone en función la primera línea

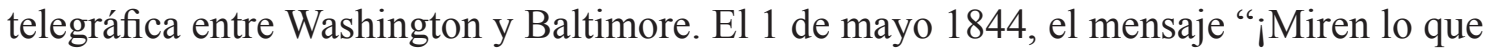
Dios ha hecho!" llega exitosamente a la estación de Mount Clare. La posibilidad técnica de la comunicación a distancia se hace posible y, entre el 1866 y 1878, el barco a vapor SS Great Eastern instala alrededor de 48.000 kilómetros de cables transoceánicos conectando Inglaterra con Canadá e India (Hartmann 2014) Ahora, lo que llama la atención de Kapp respecto a estos acontecimientos no es simplemente la posibilidad de intercambiar información entre lugares distantes, sino la misma estructura interna de los cables (ver Fig. 2). Kapp recuerda el patólogo alemán Rudolf Virchow, el cual en una ponencia dedicada a la anatomía y fisiología de la medula espinal, había observado que la estructura interna de un nervio se parece a la estructura interior de un cable telegráfico submarino: "De hecho - declaraba Virchow - los nervios son los cables del cuerpo animal así como los cables telegráficos son los nervios de la humanidad" (Virchow, citado en Kapp 2018, 104). Uno de los aspectos más interesantes de las metáforas virchowianas es la sugerencia que con la construcción de los cables transcontinentales nos proporciona conocimientos respecto a la relación entre escultura y función de los nervios. El parecido entre los nervios y los cables telegráficos no llamó solo la atención a Virchow.

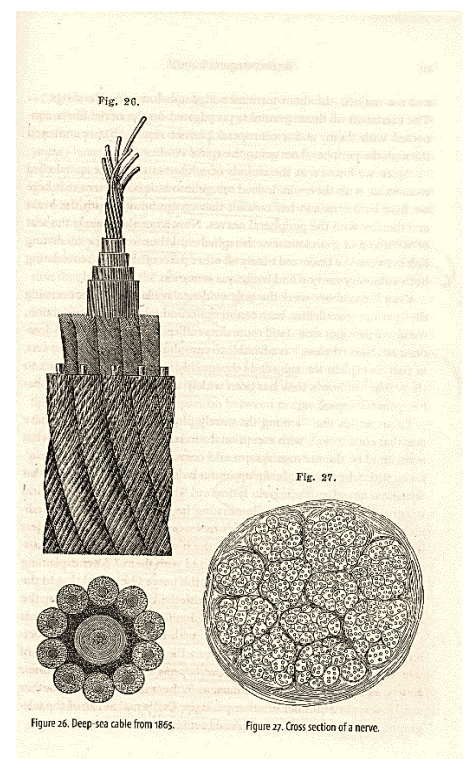

Fig. 2. Comparación entre un cable submarino y una sección de un nervio (Kapp 2018, 105). 


\section{En el principio era la mano: Ernst Kapp y la relación entre máquina y organismo Maurizio Esposito}

Kapp también recuerda el filósofo natural Carl Gustav Carus, que encontró una coincidencia entre las fibras nerviosas y los cables conductores de un telégrafo "galvánico" (Telégrafo electromagnético). En este sentido, un telégrafo galvánico adquiere el estatus de dispositivo epistémico en la medida que nos puede ayudar a entender el funcionamiento del sistema nervioso. Lo sorprendente es que Kapp olvida de mencionar el trabajo de William Thomson (Lord Kelvin) "On the Theory of Electric Telegraph" del 1855, donde el matemático ingles desarrolla la teoría matemática útil para las instalaciones de los cables transoceánicos. Esta teoría está a la base de la así llamada ecuación del cable que describe y calcula el flujo de la corriente en las dendritas y axones (Holmes 2014). Una vez más, no es el sistema nervioso que se puede reducir o describir en términos mecánicos, sino que es la tecnología telegráfica que revela puntos de contactos significativos con el sistema nervioso. La relación entre las fibras nerviosas y los cables del telégrafo es "organocéntrica" y no "mecanocéntrica".

El último ejemplo que quiero mencionar para esclarecer la teoría de la proyección orgánica se refiere a algunas infraestructuras arquitectónicas. De hecho, para Kapp, hay muchas similitudes interesantes entre la arquitectura de los puentes de armadura (truss bridges) y la estructura ósea. La función que ambas estructuras tienen que satisfacer son similares: sustentar pesos y resistir a distintas solicitudes externas. Kapp menciona el anatomista y cirujano alemán Julius Wolff (1836-1902) el cual había identificado una clara correlación entre la conformación ósea y las tenciones y compresiones que esta misma conformación debía tolerar. La ley de Wolff, de hecho, supone que la misma forma y desarrollo de la estructura ósea responde a las solicitudes físicas y mecánicas externas. En otras palabras, la morfología de un fémur humano, que es el objeto de estudio de Wolff, depende de factores externos que lo moldean de acuerdo a las tenciones y compresiones externas. Esto significa que la distribución de las líneas de tención y de compresión — que el mismo Wolff podía observar cortando el hueso en láminas delgadas - seguía en las solicitudes físicas que el mismo hueso había sufrido durante su desarrollo. La estructura, por tanto, depende y se origina a partir de la función de una manera parecida a la morfología de un puente. Para Wolff era evidente que la naturaleza construyó los huesos como un ingeniero construye un puente (Kapp 2018, 87). De acuerdo a la teoría de la proyección orgánica, existe una relación entre las soluciones que la naturaleza encuentra para resolver problemas específicos y las soluciones que los seres humanos encuentran para resolver problemas similares. Kapp explica la interrelación entre estructura mecánica y orgánica (huesos) postulando dos movimientos esenciales: por un lado tenemos,

[...] un desplazamiento hacia el exterior del cuerpo en su "imagen residual" material (after image) y por el otro lado tenemos la aplicación inversa del mecanismo para entender el organismo, incrementando la consciencia respecto a la conjugación inconsciente de un interior y exterior...la estructura esponjosa de la substancia ósea, hueso esponjoso, ha siempre sido a la vista. Los hombres primitivos lo conocían como los anatomistas modernos; en cortando el hueso en forma longitudinal con un hacha para sacar la medula. $(2018,89)$

Revista de Humanidades de Valparaíso, 2019, No 14, 117-138

(c) $(1)(9)$ CC BY-NC-ND 


\section{En el principio era la mano: Ernst Kapp y la relación entre máquina y organismo Maurizio Esposito}

Paradójicamente, entendemos la estructura interna de un hueso construyendo un puente en la medida que este último, como "imagen residual" material, puede funcionar como dispositivo epistémico para comprender algunos principios básicos que caracterizan la estructura ósea. También en este ejemplo, que probablemente es el más especulativo y débil de Kapp, se desprende una línea argumentativa bastante sofisticada: la relación entre máquina y organismo no es simplemente metafórica, sino que es al mismo tiempo práctica y epistémica. La relación es practica porque la proyección orgánica - y por tanto el lazo que se establece entre artefacto, máquinas - requiere una interacción constante, inconsciente o consciente, con el medio. No hay proyección sin una manipulación reiterada con el mundo externo y, por tanto, no hay relación entre máquina y organismo sin praxis (que sea un aparato óptico respecto a los ojos, la máquina a vapor respecto a la cinemática orgánica, que sean los cables telegráficos respectos al sistema nervioso o un puente en correspondencia con la estructura ósea). La relación es epistémica en virtud del hecho que un mecanismo surge a partir de una práctica que es, antes inconsciente y luego, a partir de sus productos, consciente. El conocimiento del mundo orgánico depende literalmente del conjunto de artefactos y máquinas que los seres humanos han ido construyendo desde el inicio de la historia, es decir, a partir de la construcción de los primeros utensilios?.

Antes de pasar a la siguiente sección, nos queda una tarea importante. Hemos visto que un aspecto fundamental de la teoría de loa proyección orgánica es la producción inconsciente de lo que Kapp llama "imagen residual" material, es decir, la construcción del órgano/herramienta que mantiene sus lazos miméticos con el organismo. Sin embargo, ¿Qué se entiende con "inconsciente”? ¿En qué sentido la producción de un artefacto es "inconsciente”? Y, ¿Por qué Kapp entiende el fenómeno de la proyección orgánica como una dialéctica entre inconsciente y consciente? Antes que todo, hay que recordar que Kapp escribe antes de Freud, por tanto su concepción de "inconsciente" no tiene ninguna relación con la concepción Freudiana. El referente principal de Kapp es el referente de caso todos los que escribieron sobre el inconsciente en el siglo XIX y XX (incluso el mismo Freud), es decir, el filósofo alemán Eduard von Hartmann y su texto Philosophie des Unbewußten (Filosofía del inconsciente) publicado al 1869 y traducido en ingles en 1884. El punto inicial de la reflexión de Hartmann es una breve sección de la Antropología de Kant: "Sobre las ideas que tenemos sin ser conscientes de ella" (von Hastmann 1884, Cap. I). En la sección Kant expresa muy claramente el dominio de reflexión que debe interesar el filósofo del inconsciente. Aunque la palabra Unbewusste todavía no existía, Kant sostuvo que: "...lo cierto es que podemos ser mediatamente conscientes de tener una representación, aun cuando no seamos inmediatamente conscientes de ella. Este

\footnotetext{
${ }^{7}$ Es una lástima que Kapp no haya podido asistir al desarrollo de la industria aeronáutica. Pocos años después de la muerte de Kapp en 1896, los hermanos Wright y el inventor brasileño Alberto Santos Dumont construían los primeros prototipos de aeroplanos. Muy probablemente, Kapp hubiera considerado el avión como una proyección orgánica de un ave, y, luego, un dispositivo epistémico que nos entrega conocimientos de primera mano sobre los principios del vuelo.
}

Revista de Humanidades de Valparaíso, 2019, No 14, 117-138

(c) $(1)(9)$ CC BY-NC-ND 
En el principio era la mano: Ernst Kapp y la relación entre máquina y organismo Maurizio Esposito

género de representaciones se llama, entonces, obscuras; las restantes son claras" (Kant 2014, 19). Lo que Kant entiende con obscuro o claro, mediato o inmediato tiene una relación con lo que los psicólogos de la Gestalt llamaban cosificación, es decir, la facultad de completar, añadir elementos o formas a percepciones fragmentarias con la diferencia esencial que para Kant lo que no percibimos inmediatamente los percibimos en forma no consciente:

Cuando se es consciente de estar viendo a lo lejos, en una pradera, un hombre, si bien no se es consciente de ver sus ojos, nariz, boca, etc., propiamente se concluye solo que aquella cosa es un hombre; pues sí, porque no se es consciente de percibir estas partes de la cabeza (e igualmente las restantes partes de ese hombre), se quisiera sostener que no se tiene en absoluto en la intuición la representación de ellas, tampoco se podría decir que se ve un hombre; pues de estas representaciones parciales está compuesta la total (de la cabeza o del hombre). $(2014,19)$

Lo "inconsciente" kantiano, entonces, se refiere a todas aquellas sensaciones que no alcanzan a formar una representación clara (consciente). Y, como después notaran todos los más importantes pensadores de los inconscientes, Kant también enfatiza el hecho que el dominio de las sensaciones inconscientes es mucho mayor que el dominio de lo consciente:

El hecho que sea inmenso el campo de aquellas nuestras intuiciones de los sentidos y las sensaciones de las que no somos conscientes; si bien podemos concluir indubitablemente que las tenemos; esto es, de las representaciones obscuras en el hombre (y también en los animales); de que las claras, por el contrario, encierren solo unos, infinitamente pocos, puntos de aquellos que están abierto a la consciencia $[\ldots](2014,20)$

Por supuesto, Hartmann desarrolla la intuición básica de Kant dividiendo su análisis entre la manifestación del inconsciente en el cuerpo y en la mente humana hasta proponer - como sugiere el mismo Kapp - una "modesta" teoría del universo (Kapp 1870). Sin embargo, con la excepción de la reseña que dedicó a Hartmann en 1870, Kapp no profundiza mucho el asunto en relación a su teoría de la proyección orgánica. Mencioné Hartmann, y especialmente me detuve sobre Kant, para aclarar lo que probablemente asumía Kapp cuando escribe de "inconsciente" en relación a la proyección orgánica. De hecho, con "inconsciente" no debemos imaginar algo místico o vago sino algo bastante simple: las primeras interacciones de los seres humanos con su ambiente son interacciones prácticas que generan un conjunto de saberes, creencias, actitudes y enfoques que son inicialmente "obscuras", es decir, no existe una representación o teoría "clara" respecto a lo que se hace y como se hace. La distinción Ryleiana entre "saber cómo" y "saber que" es una buena aproximación a la diferencia Kappiana entre conocimiento inconsciente y consciente (Ryle 1945). La práctica que establece una primera vinculación con el medio construyendo artefactos es algo inconsciente (un tipo de saber cómo). Esta misma prác-

Revista de Humanidades de Valparaíso, 2019, No 14, 117-138

(c) $(9)(9)$ CC BY-NC-ND 
En el principio era la mano: Ernst Kapp y la relación entre máquina y organismo Maurizio Esposito

tica se vuelve consciente cuando el sujeto reconoce "claramente" que el artefacto es una proyección orgánica de un órgano/herramienta. El artefacto es el medio que conduce al sujeto a producir un "saber qué", o una teoría o representación, respecto al mundo orgánico. En síntesis, la práctica, por un lado, es intuitiva, eficiente respecto a sus objetivos $\mathrm{y}$, por la mayor parte, obscura respecto a sus representaciones (inconsciente). El conocimiento teórico, adquirido por medio de esta práctica, es reflexivo, consciente y, por la mayor parte claro, en referencia a sus representaciones. De acuerdo a este esquema, la proyección orgánica es el resultado de un movimiento dialectico entre practica (elemento inconsciente - obscuro) y teoría (elemento consciente - claro) así que podemos tener representaciones claras sobre los orgánico cuando ya hemos interiorizado, en forma inconsciente, las prácticas que nos han llevado a producir un artefacto o mecanismo que es, en realidad, una proyección de lo orgánico.

\section{Desde la Bio-filosofía a la Tecno-filosofía}

Unas de las implicancias de la filosofía de Kapp — así como todas las filosofías que ven la tecnología como una proyección, directa o indirecta, de lo orgánico- es que hay una relación constitutiva e imprescindible entre máquina y organismo. Esto no significa defender, como hemos visto, que un organismo es nada más que una máquina o que puede ser reducido, es sus elementos principales, a un artefacto mecánico. El argumento es más sofisticado: la relación mecanocéntrica que podemos reconocer es solo el producto de un proceso dialectico que involucra la historia, la ontología y la práctica. Una máquina se parece a un organismo no porque subsiste una identidad evidente y/o obvia entre los dos, sino porque un mecanismo es una proyección orgánica del organismo mismo. Es en virtud de esta inversión del argumento que podemos identificar las afinidades y diferencias entre máquinas y organismos. En distintas ocasiones, Kapp sostiene que no podemos confundir una máquina con un órgano u organismo porque: "El organismo, como todo el mundo natural, es un devenir mientras que el mecanismo es algo terminado. En el primero hay desarrollo y vida, en el segundo hay solo composición e inercia. Los que no comparten esta diferencia no entienden la distinción entre un sacacorchos que llevan en el bolsillo y su muñeca como un participante integral de una actividad orgánica auto motivada" (Kapp 2018, 49-50). Lo que nos sugiere Kapp es que, por un lado y desde una perspectiva ontológica, el organismo no es un mecanismo y tampoco puede ser reducido a este. Por el otro lado, el mecanismo si puede entregarnos conocimientos sobre el mundo orgánico, en virtud de su estatus de proyección o mimesis orgánica. En suma, la máquina y organismo son dos entidades ontológicamente distintas, pero tienen una relación epistémica heurísticamente productiva.

Si excluimos la idea que haya una continuidad ontológica entre mecanismos y mundo orgánico (así como no hay una continuidad ontológica entre el sujeto de un retrato y el retrato mismo), es evidente que la pregunta filosófica interesante no es si un organis-

Revista de Humanidades de Valparaíso, 2019, No 14, 117-138

(c) (i) $(9)-1$ CC BY-NC-ND 
En el principio era la mano: Ernst Kapp y la relación entre máquina y organismo Maurizio Esposito

mo sea realmente un mecanismo (no puede serlo de acuerdo a lo anterior). De acuerdo a la perspectiva "órganocéntrica", la pregunta filosóficamente pertinente es: ¿Qué tipo de relaciones subsisten entre prácticas, artefactos, máquinas y nuestra comprensión del mundo orgánico? El problema que debemos enfrentar es entonces epistémico y se refiere al paralelismo histórico y cognitivo entre determinadas tecnologías y representaciones (objetos e ideas). Dada lo anterior, podemos reinterpretar de una manera "organocéntrica" la visión de los cartesianos que identificaban los animales con los autómatas complejos. Y podemos hacer lo mismo con Helmholtz o Meyer, que veían el cuerpo humano como un tipo de máquina a vapor. Con Virchow y Carus, que veían el sistema nervioso como un conjunto de cables telegráficos. Con Wolff, que veía un fémur como un ejemplo de puente de armadura. La lista podría bien continuar hasta nuestros días. Podemos incluir, entre otros, a Norbert Wiener, que veía un organismo como un mecanismo cibernético. A James Watson and Francis Crick, que veían la secuencia de las bases de ADN y sus productos (cadena de aminoácidos) como un código basado en cuatro "letras" ". Hoy en día no faltan científicos que comparan el genoma a un programa informático y el cerebro a un computador. La interpretación que normalmente se ofrece para explicar esta relación entre entidad orgánica y artefacto tecnológico es "mecanocéntrica". Simplemente se asume que el genoma o el cerebro tienen una conexión privilegiada con las tecnologías de la información porque los principios de funcionamiento del genoma y cerebro son mecánicos. ${ }^{9}$ Sin embargo, se reinterpretamos dicha conexión en términos "organocéntricos", un nuevo espacio de investigación muy interesante se abre para los bio-filósofos y tecno-filósofos en conjunto. Y este espacio consistiría en explorar y averiguar cómo nuevas tecnologías crean las condiciones para comprensión del mundo orgánico sin que este último se reduzca a las primeras.

En las últimas décadas, la proliferación de entidades híbridas no ha hecho que consolidar la intuición de Kapp que haya una proyección contante de lo orgánico a lo mecánico, con la diferencia que la distinción entre máquina y organismo se puede esfumar con la creación de un organismo sintético. Con los avances tecnológicos, la proyección se puede trasformar en identidad ontológica en el momento que organismos sintéticos pueden producir otros organismos sintéticos (es decir, máquinas/organismos). Por supuesto, dicha identidad ontológica todavía no se ha cumplido y es material de especulación respecto a cuándo será posible realizarla. Empero, la tecnología actual hace esta posibilidad muy probable en las próximas décadas. La proyección orgánica, es decir la imagen residual,

\footnotetext{
${ }^{8}$ Es evidente que la metáfora del "código" presupone la existencia de alguna tecnología de la información.

${ }^{9}$ Incluso los que cuestionan la relevancia o la pertinencia de las metáforas mecanicistas, no pueden negar su fuerza y utilidad, ahora como antes. Después de todo, identificar las diferencias entre máquina y organismo no necesariamente implica descalificar el mecanismo, sino usarlo como marco heurístico que nos permite reconocer algunos elementos únicos e irreducibles de los organismos mismos. Los elementos de continuidad y discontinuidad entre máquinas y organismos nos proporcionan igualmente conocimientos positivos sobre ambos.
}

Revista de Humanidades de Valparaíso, 2019, No 14, 117-138 
En el principio era la mano: Ernst Kapp y la relación entre máquina y organismo Maurizio Esposito

puede aproximarse a tal punto a un organismo que la proyección misma coincidiera con una verdadera reproducción. Podemos de hecho sugerir que hay "proyección" cuando falta una identidad ontológica entre entidad proyectante y entidad proyectada mientras que, por lo contrario, hay "reproducción" cuando existe una continuidad ontológica entre entidad reproductora y entidad reproducida. Este es, sin embargo, un tema que nos llevaría muy lejos respecto a los objetivos de este artículo.

Lo que todas estas observaciones nos pueden sugerir es que las reflexiones filosóficas sobre lo orgánico no pueden prescindir de reflexiones filosóficas sobre la tecnología. Hasta al momento, las interacciones entre filosofía de la tecnología y filosofía de la biología han sido muy esporádicas. Sin embargo, un aspecto importante de la hipótesis de la proyección orgánica es hacer esta interacción necesaria y productiva. Si los artefactos tecnológicos son una proyección de lo orgánico y si, viceversa, estos mismos artefactos se vuelven dispositivos epistémicos para entender lo orgánico, entonces la bio-filosofía y la tecno-filosofía deben complementarse. Desde un punto de vista histórico, cada tecnología ha creado las condiciones para nuevas posibilidades de representación y comprensión. Nos relacionamos con el mundo por medio de artefactos y por medio de estos artefactos también testeamos y comprendemos el medio que nos rodea. Por supuesto, no hay que olvidar que la relación entre máquina y artefacto es dinámica y variable. No es lo mismo entender un organismo como un autómata, una máquina a vapor, un sistema cibernético o un computador. Algunos artefactos mecánicos pueden imitar algunos aspectos estructurales, otros se refieren a ciertos otros elementos funcionales. Cada tecnología puede revelar propiedades distintas de la organización biológica, así como distintos sistemas experimentales revelan dominios específicos, y a veces complementares, de realidad.

Podemos decir entonces que la postura "organocéntrica" nos proporciona una perspectiva bastante más sofisticada de la postura "mecanocéntrica". Defender la idea que un organismo (o un órgano) se reduce a un mecanismo (o una máquina) es, como mínimo, una extrema simplificación por dos razones principales. En primer lugar, no hay la Máquina en general, así como no hay la Tecnología en general. Hay distintas máquinas que funcionan con tecnologías diferentes y que proporcionan miradas alternativas del mundo orgánico (ej. tecnologías mecánicas, termodinámicas o electromagnéticas). En segundo lugar, incluso si pudiéramos llegar a definir en términos muy generales - como intentó hacerlo Franz Reuleaux (Moon 2007), algunas de las características esenciales que una entidad debe necesariamente poseer para ser una máquina, estas propiedades serán muy probablemente propiedades orgánicas "proyectadas" en un artefacto tecnológico. Suponiendo que haya una definición univoca de máquina (o de mecanismo), dicha definición no nos serviría para reducir propiedades orgánicas a propiedades mecánicas por la razón que la representación de máquina presupondría una representación previa de organismo (Canguilhem 2000) ${ }^{10}$. Los artefactos más simples o los aparatos tecnológicos más sofisti-

${ }^{10}$ De hecho, Canguilhem sostenía la aparente paradoja segundo la cual no existe nada de más orgánico de

Revista de Humanidades de Valparaíso, 2019, No 14, 117-138

(c) $(9)(9)$ CC BY-NC-ND 
En el principio era la mano: Ernst Kapp y la relación entre máquina y organismo Maurizio Esposito

cados son imitaciones de la naturaleza y, como vimos en la primera sección del artículo, el primer modelo imitado es el mismo cuerpo humano. El brazo y la mano son el prototipo del martillo y el martillo es el prototipo de todos los artefactos que siguen. La empresa tecnológica, para Kapp, se basa sobre un bio-mimetismo inconsciente. Si aceptamos esta premisa tenemos que concluir que, en realidad, cualquier máquina o mecanismo es resultado de una imitación originaria y esta imitación puede interesar aspectos estructurales (organización de la máquina) o funcionales (actividades de la máquina).

Dado todo lo anterior, entendemos que la relación "organocéntrica" consiste precisamente en sostener que las máquinas son un subconjunto del conjunto del mundo orgánico. La discusión, por tanto, se desplaza desde una conexión sincrónica (si las máquinas representan fielmente a los organismos) a una conexión diacrónica (como las máquinas llegan a constituirse como dispositivos epistémicos en relación con los fenómenos orgánicos). De acuerdo a la hipótesis "organocéntrica", de hecho, la identidad entre máquinas y organismos no subsiste aunque cuando las primeras nos proporcionen conocimientos genuinos en biología. Un acercamiento a la filosofía de la tecnología de parte de los bio-filósofos, y viceversa, nos puede ayudar a entender mucho mejor las relaciones entre máquinas y organismos. Este acercamiento es hoy en día particularmente importante para entender como las nuevas tecnologías informáticas globales representan, definen y plasman nuestras concepciones del mundo orgánico (Stevens 2011). En suma, la hipótesis "organocéntrica" de Kapp hace la conjunción entre tecnología y biología imprescindible en la medida que la primera es un derivado de la segunda y la segunda se vuelve inteligible a partir de la primera.

\section{Conclusiones}

En arrojando luz sobre un autor poco conocido, el articulo ha explorado un enfoque alternativo sobre la relación entre máquina y organismo. En primer lugar, he sugerido que podemos diferenciar dos maneras principales de articular dicha relación. Por un lado, hay una manera tradicional que he llamado relación "mecanocéntrica". Este consiste en sugerir que existe un cierto grado de isomorfismo entre máquina y organismo y, por tanto, la máquina (y el mecanismo) puede ser principio de explicación del mundo orgánico. He argumentado que en la relación "mecanocéntrica" el mecanismo es anterior y primordial respecto al mundo orgánico en razón de que un organismo es un tipo de mecanismo y no lo contrario. En la historia de la relación entra máquina y organismo, esta postura ha sido

una máquina. Esto porque la máquina es por Canguilhem una "imagen residual” del organismo. En otras palabras, podemos considerar una máquina como una "caricatura" de un organismo en la medida que la “caricatura (máquina) exagera algunos de los elementos esenciales que caracterizan un ser orgánico: en una máquina todas las piezas tienen una función específica y todas contribuyen para que el conjunto trabaje por un fin definido (el fin del constructor). Lo anterior solo se aplica de una forma muy aproximada al mundo orgánico.

Revista de Humanidades de Valparaíso, 2019, No 14, 117-138

(c) $(9)(9)$ CC BY-NC-ND 
En el principio era la mano: Ernst Kapp y la relación entre máquina y organismo Maurizio Esposito

la más exitosa o, por lo menos, la más representativa en las discusiones filosóficas. Hemos visto que, sin embargo, existe una alternativa menos representativa, aunque no menos fundamentada: la que he denominado relación "organocéntrica". Esta consiste en suponer que las máquinas son una imitación de un organismo o sus partes. Por ende, el dominio de lo mecánico es un subconjunto del conjunto del mundo orgánico y esto implica que, a diferencia de la relación "mecanocéntrica", el organismo es primordial respecto al mecanismo en razón de que este último es solo un tipo de organismo.

Lo que he tentativamente llamado relación "organocéntrica" es una generalización ligada a la hipótesis de la proyección orgánica propuesta de Kapp y a todas las hipótesis que consideran los artefactos mecánicos como entidades biomiméticas. Hemos visto que, de acuerdo a Kapp, el proceso de proyección se puede dividir en tres momentos; en primer lugar, hay una interacción practica entre organismo (ser humano) y medio ambiente. Esta interacción práctica, la cual nace a partir de algunas necesidades adaptativas, genera las condiciones para la producción de artefactos. El segundo momento consiste en la creación de artefactos funcionales que son, en realidad, entidades biomimética. En este estadio, el proceso de proyección comienza a ser consciente, es decir, el sujeto empieza a percibir que el artefacto es una imitación orgánica que proporciona una mejor integración con el medio ambiente. Sin embargo, es solo en el tercer momento que el sujeto realiza, conscientemente, que la máquina es, en realidad, una proyección orgánica. En virtud de este reconocimiento consciente, la máquina se vuelve un dispositivo epistémico eficaz para entender los procesos orgánicos. En otras palabras, los artefactos técnicos no solamente facilitan la integración entre organismo y medio ambiente, sino que también favorecen el conocimiento del mundo externo. De acuerdo a la hipótesis de Kapp, el artefacto y luego la máquina, considerados como mediadores eficientes entre sujetos y medio ambiente, producen las condiciones para la emergencia de la consciencia (evolución del cerebro), el comienzo de la historia y civilización.

He también sugerido que la hipótesis de la proyección orgánica nos orienta hacia una integración estratégica entre bio-filosofía y tecno-filosofía. Esta integración nos permite articular una postura más sofisticada respecto a la visión "mecanocéntrica": es decir, nos lleva a defender la hipótesis que los organismos y las máquinas son entidades ontológicamente distintas, aunque cuando las últimas nos pueden entregar conocimientos genuinos y confiables sobre los segundos. Todo esto en virtud del hecho que los artefactos y las máquinas son, en esencia, una expresión del organismo, y, más en lo específico, son una derivación del órgano/herramienta primordial y prototípico: la mano. Finalmente, hemos visto que la hipótesis "organocéntrica" nos puede liberar de la discusión interminable (y probablemente sin posibilidad de solución) respecto a si los organismos son máquinas. La discusión, de hecho, está basada sobre un prejuicio "mecanocéntrico" motivado más de una intuición implícita (ej. Que las máquinas se parecen a los organismos) que de una reflexión explicita (ej. En qué sentido y como las máquinas se relacionan con los organismos). Entonces, en continuidad con la hipótesis kappiana, las tareas que nos quedan hacer

Revista de Humanidades de Valparaíso, 2019, No 14, 117-138

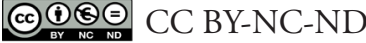


En el principio era la mano: Ernst Kapp y la relación entre máquina y organismo Maurizio Esposito

hoy en día son principalmente dos: 1) Como, y hasta qué punto, los artefactos tecnológicos han influido sobre las diferentes concepciones de vida y organismo en la historia de la biología. 2) Averiguar cómo y en qué medida las nuevas tecnologías, mucho más globales y ubicuas que las tecnologías del siglo XIX, están plasmando los nuevos conocimientos sobre el mundo de lo vivo, así como el mundo inorgánico. De hecho, nuevas formas de interacción con el mundo crean las condiciones para la generación de nuevos artefactos tecnológicos, y por tanto, nuevas representaciones. La hipótesis "organocéntrica" nos sugiere que la historia de la biología no es solamente la historia de sus ideas sino que es también (y sobretodo) la historia de artefactos que se han trasformado en dispositivos epistémicos que, a su vez, produjeron nuevas formas de mirar, interpretar, y entender los seres vivos.

\section{Agradecimientos}

Este trabajo ha sido redactado en ejecución del proyecto de investigación Fondecyt N. 1171017.

\section{Referencias bibliográficas}

Aristóteles (2003). Acerca del Alma. Madrid: Editorial Gredos.

Canguilhem, G. (2000). La connaissance de la vie. Paris: Vrin.

Duchesneau, F. (1998). Les Modèles du vivant de Descartes à Leibniz. Paris: Vrin.

Espinas, A. (1897). Les origines de la technologie: étude sociologique. Paris: Felix Alcan.

Espinas, A. (1903). L'organisation ou la Machine Vivante en Grèce, au IV siècle avant J.C. Revue de Métaphysique et de Morale, 11(6): 703-71.

Gehlen, A. (1989). Man in the Age of Technology. New York: Columbia University Press.

Hacking, I. (1998). Canguilhem amid the Cyborgs. Economy and Society, 27: 2-3, 202-216.

Hartmann, F. (2014). Of Artefacts and Organs: World Telegraph Cables and Ernst Kapp's Philosophy of Technology. En W. Boyd Rayward (ed.), Information beyond Borders. Burlington: Ashgate.

Heudin, J. (2008). Les Créatures artificielles: Des automates aux mondes virtuels. Paris: Odile Jacob.

Holmes, W.L. (2014). Cable Theory: Overview. En D. Jaeger, R. Jung (eds.), Encyclopedia of Computational Neuroscience. Springer, New York, NY.

Kant, I. (2014). Antropologia en Sentido Pragmatico. México: Fondo de Cultura Económico.

Kapp, E. (1849). Der konstituierte Despotismus und die konstitutionelle Freiheit. Hamburg: Hoffmann und Campe.

Revista de Humanidades de Valparaíso, 2019, No 14, 117-138

(c) $(1) @(9)$ CC BY-NC-ND 
En el principio era la mano: Ernst Kapp y la relación entre máquina y organismo Maurizio Esposito

Kapp, E. (1870). Essay review: Philosophy of the Unconscious. Journal of Speculative Philosophy, 4(1): 84-94.

Kapp, E. (1877). Grundlinien einer Philosophie der Technik. Braunschweig: Druck und Verlag von George Westermann.

Kapp, E. (2018). Elements of a Philosophy of Technology: On the Evolutionary History of Culture. Minneapolis: University of Minnesota Press.

Mitcham, C. (1994). Thinking through Technology: The Path between Engineering and Philosophy. Chicago: Chicago University Press.

Moon, F. (2007). Franz Reuleaux: Contributions to 19th Century Kinematics and Theory of Machines. Appl. Mech. Rev., 56(2): 261-285.

Nicholson, D. (2012). The concept of mechanism in biology. Studies in History and Philosophy of Biological and Biomedical Sciences, 43: 152-163.

Riskin, J. (ed.) (2007). Genesis Redux: Essays in the History and Philosophy of Artificial Life. Chicago: Chicago University Press.

Ryle, G. (1945). Knowing How and Knowing That: The Presidential Address. Proceedings of the Aristotelian Society, 46: 1-16.

Ortega y Gassett, J. (1965). Meditación de la Técnica. Madrid: Espasa-Calpe.

Simondon, G. (2012). Du mode d'existence des objets techniques. Paris: Aubier.

Spengler, O. (2015). Man and Technics: A Contribution to a Philosophy of Life. London: Arktos Media.

Stevens, H. (2011). Life out of sequence: a data-driven history of informatics. Chicago: Chicago University Press.

von Hartmann, E. (1884). Philosophy of the Unconscious. London: Trubner \& Co.

Wood, G. (2002). Living dolls: a magical history of the quest for mechanical life. London: Faber and Faber. 